PO-0302 PROGNOSTIC FACTORS AND OUTCOME OF TRAUMATIC BRAIN INJURY IN CHILDREN TREATED IN PICU

${ }^{1}$ A Violaki, ${ }^{1} \mathrm{~K}$ Salpigidou, ${ }^{2} \mathrm{D}$ Charalampopoulos, ${ }^{3} \mathrm{E}$ Hochliourou, ${ }^{4} \mathrm{E}$ Kiritsi, ${ }^{3} \mathrm{M}$ Dimitriadou, ${ }^{3} \mathrm{M}$ Sdouga. ${ }^{1} \mathrm{PICU}$, Hippokration General Hospital, Thessaloniki, Greece; ${ }^{2}$ University College, University College, London, UK; ${ }^{3}$ PICU, Hippokratio General Hospital, Thessaloniki, Greece; ${ }^{4}$ Nursing, Educational Institute, Athens, Greece

\subsection{6/archdischild-2014-307384.953}

Background Prediction of outcome based on certain prognostic factors is still challenging in paediatric traumatic brain injuries (TBI). Purpose of our study is to evaluate prognostic factors and outcome in children with TBI admitted in PICU.

Methods The sample was 252 children with TBI who were admitted to PICU of Hippokratio General Hospital of Thessaloniki from 1999 to 2012. The statistical analysis was performed with the statistical package SPSS 17 and the application of statistical tests $\mathrm{x}^{2}$-test, anova, Mann Whitney test, t-test and Pearson Correlation. Age, sex, Glasgow Coma Scale (GCS), Paediatric Risk Index of Mortality (PRISM score), days of mechanical ventilation, hypotension and need of inotropic support as well as late hyperglycemia were correlated to outcome.

Results Age groups, sex and mechanism of injury showed no correlation to outcome. Patients who died had statistically significant higher mean PRISM score $(25,7 \pm 8,4)$,compared with patients with good outcome $(7,5 \pm 4,7)$. The application of Pearson Correlation showed that the increased rating of PRISM score, as well as the low grading GCS were positively correlated to death, late hyperglycemia, haemodynamic instability, use of inotrops, poor outcome and disabilities ( $<<0,001)$.

Conclusion A high PRISM score, a low GCS, hypotension and hyperglycemia after the first $48 \mathrm{~h}$, are directly associated with poor outcome and death. Knowledge of these prognostic factors may help neurocritical care specialists to improve outcome in paediatric TBI.

\section{PO-0303 TRAUMATIC BRAIN INJURY IN PICU}

${ }^{1} \mathrm{~A}$ Violaki, ${ }^{1} \mathrm{~K}$ Salpigidou, ${ }^{1} \mathrm{E}$ Volakli, ${ }^{2} \mathrm{E}$ Kiritsi, ${ }^{3} \mathrm{~S}$ Kalamitsou, ${ }^{3} \mathrm{E}$ Michalopoulou. ${ }^{1} \mathrm{PICU}$, Hippokration General Hospital, Thessaloniki, Greece; ${ }^{2}$ Nursing, Educational Institute, Athens, Greece; ${ }^{3}$ PICU, Hippoktation General Hospital, Thessaloniki, Greece

\subsection{6/archdischild-2014-307384.954}

Background Traumatic brain injury (TBI) still remains the most common cause of morbidity and mortality in children all over the world. The purpose of this study is to specify the demographic and epidemiologic characteristics of all TBI cases treated in the PICU of Hippokratio General Hospital of Thessaloniki, over a 12-year period.

Methods A retrospective chart review was conducted of all patients admitted to PICU from 1999 to 2012 with TBI. Age, sex, mechanism and severity of injury, Glasgow Coma Scale (GCS), Paediatric Risk Index of Mortality (PRISM) and outcome were recorded.

Results TBI cases accounted for 14,5\% of total admissions (252 patients). The mean age was $6,9 \pm 3,6$ with a predominance of boys $69,4 \%$. The most common cause of injury was falls $(34,9 \%)$, followed by car accidents $(33,7 \%)$ and pedestrian road accidents $(19,8 \%)$. A total of $71 \%$ were classified as severe TBI $($ GCS $<8)$. Mean PRISM score was 10,6 $\pm 7,9$. The rate of operative intervention was $33 \%$. Good recovery was observed in $68,7 \%$, poor recovery in $20,6 \%$ while death in $10,7 \%$.
Conclusion TBI consists a common cause of admission in PICU and despite the advances in medical and surgical care remains the leading cause of death and disability for children in all age groups expect from infants $<1$ year of age. The majority of TBI, in our series, were severe in nature and almost one third of them resulted in severe disability or death.

\section{P0-0304 SAFETY OF THE MANCHESTER TRIAGE SYSTEM IN PAEDIATRIC PATIENTS: DOES IT RECOGNISE THE CRITICALLY ILL?}

${ }^{1} \mathrm{JM}$ Zachariasse, ${ }^{2} \mathrm{JW}$ Kuiper, ${ }^{1} \mathrm{HA}$ Moll, ${ }^{1} \mathrm{M}$ van Veen. ${ }^{1}$ General Paediatrics, Erasmus MC Sophia Children's Hospital, Rotterdam, Netherlands; ${ }^{2}$ Paediatric Intensive Care, Erasmus MC Sophia Children's Hospital, Rotterdam, Netherlands

\subsection{6/archdischild-2014-307384.955}

Background Triage is an important method to manage patient flows safely at the Emergency Department (ED), by prioritising patients according to their clinical urgency. To date, no studies have assessed the safety of the Manchester Triage System (MTS) to identify critically ill children.

Objective To determine how well the MTS identifies critically ill children as reflected by ICU admission, and to identify areas for improvement.

Methods In this observational study, we collected routinely available electronic data of all children $<16$ years visiting the ED in a Dutch university hospital between 2006 and 2012. Under triage was defined as patients triaged to low MTS urgencies (level 3 to 5, indicating 60, 120 and 240 min maximum waiting time) who were admitted to ICU. Patients with low MTS urgencies and ICU-admission were compared with low urgency patients who were not admitted to ICU, to identify risk factors for under triage.

Results During the 7-year study period, data on 44476 ED-visits were collected of which $837(1,9 \%)$ were from patients admitted to ICU. Of all ICU admissions, 251 (28.3\%) were assigned to low MTS triage categories. Risk factors for under triage were young age, non-surgical presenting problem and general illness, gastrointestinal or respiratory complaints (all $\mathrm{p}<$ 0.001). Moreover, under triaged children were more often referred by a medical specialist, possibly indicating that co morbidity is a risk factor too.

Conclusion The MTS under triages an impressive amount of paediatric patients. Adaptations of the MTS based on risk factors for under triage are needed to improve the safety of the MTS.

\section{PO-0305 THREE CASES OFF PRES-POSTERIOR REVERSIBLE ENCEPHALOPATHY SYNDROME}

LM Silva, SH Victal, F Lobo, F Capela, BES Araujo. Cti Pediatrico, Inca, Rio de Janeiro, Brazil

\subsection{6/archdischild-2014-307384.956}

PRES is characterised by headache, seizures, nausea, vomiting and visual disturbance due to severe hypertension. The clinical course is reversible without neurologic sequel after hypertension has been controlled.

A four-year-old boy, with Lymphoblastic Lymphoma. He was in course of hydrocortisone in high doses-20 mg/Kg/day, and developed Hypertension, focal seizures and bilateral amaurosis. We treated the hypertension with captopril, furosemide and 